\title{
Black holes in pseudobulges: demography and models
}

\author{
F. Shankar ${ }^{1}$, F. Marulli ${ }^{2,3,4}$, S. Mathur ${ }^{5,6}$, M. Bernardi ${ }^{7}$, and F. Bournaud ${ }^{8}$ \\ ${ }^{1}$ GEPI, Observatoire de Paris, CNRS, Univ. Paris Diderot, 5 Place Jules Janssen, 92195 Meudon, France \\ e-mail: francesco.shankar@obspm.fr \\ 2 Dipartimento di Astronomia, Universitá degli Studi di Bologna, via Ranzani 1, 40127 Bologna, Italy \\ 3 INAF - Osservatorio Astronomico di Bologna, via Ranzani 1, 40127 Bologna, Italy \\ 4 INFN/National Institute for Nuclear Physics, Sezione di Bologna, viale Berti Pichat 6/2, 40127 Bologna, Italy \\ 5 Department of Astronomy, Ohio State University, McPherson Laboratory, 140 W. 18th Ave., Columbus, OH 43210-1173, USA \\ ${ }^{6}$ Center for Cosmology and Astropharticle Physics, Ohio State University, Columbus, OH 43210, USA \\ 7 Department of Physics and Astronomy, University of Pennsylvania, 209 South 33rd St, Philadelphia, PA 19104, USA \\ ${ }^{8}$ CEA, IRFU, SAp, 91191 Gif-sur-Yvette, France
}

Received 3 November 2011 / Accepted 30 January 2012

\begin{abstract}
Context. There is mounting evidence that a significant fraction of black holes (BHs) today live in late-type galaxies, including bulgeless galaxies and those hosting pseudobulges, and are significantly undermassive with respect to the scaling relations followed by their counterpart BHs in classical bulges of similar stellar (or even bulge) mass.

Aims. Here we discuss the predictions of two state-of-the-art hierarchical galaxy formation models in which BHs grow via mergers and, in one, also via disc instability. Our aim is to understand if the wealth of new data on local BH demography is consistent with standard models.

Methods. We follow the merger trees of representative subsamples of BHs and compute the fractional contributions of different processes to the final $\mathrm{BH}$ mass.

Results. We show that the model in which BHs always closely follow the growth of their host bulges, also during late disk instabilities (i.e., bars), produces too narrow a distribution of BHs at fixed stellar mass to account for the numerous low-mass BHs now detected in later-type galaxies. Models with a looser connection between BH growth and bar instability instead predict the existence of a larger number of undermassive BHs, in better agreement with the observations.

Conclusions. The scatter in the updated local $\mathrm{BH}$-bulge mass relation (with no restriction on galaxy type) appears to be quite large when including later-type systems, but it can still be managed to be reproduced within current hierarchical models. However, the fuelling of BHs during the late bar-instability mode needs to be better quantified/improved to properly fit the data. We conclude discussing how the possibly large number of $\mathrm{BHs}$ in later type galaxies demands for an in-depth revision of the local $\mathrm{BH}$ mass function and its modelling.
\end{abstract}

Key words. galaxies: bulges - galaxies: evolution - galaxies: nuclei - galaxies: statistics - galaxies: structure - cosmology: theory

\section{Introduction}

There is evidence in the local Universe that most bulgedominated galaxies seem to obey rather tight correlations between the central black holes' (BHs) masses and the bulge masses and velocity dispersions of their hosts (e.g., Magorrian et al. 1998; Ferrarese \& Merritt 2000).

Such tight correlations have been physically interpreted as byproducts of some $\mathrm{BH}$ self-regulation mechanism - active galactic nuclei (AGN) feedback - operating during the highredshift, gas-rich phases of galaxy build-up. At early times in fact, large amounts of gas inflows can be generated due to, e.g., merger events or internal instabilities (e.g., Barnes 1992; Bournaud et al. 2011b), triggering star formation and fuelling the central $\mathrm{BH}$ that can energetically and kinematically backreact on the gas reservoirs via AGN winds. Such interactions can possibly contribute to shut-down starformation and to the formation of the progenitors of today's early-type galaxies (e.g., Sanders et al. 1988; Granato et al. 2006; Shankar et al. 2008; Lapi et al. 2011).

Mounting empirical evidence suggests, however, that other additional tracks may have significantly contributed to
$\mathrm{BH}$ formation and growth. Different observations now question that $\mathrm{BH}$-host correlations indeed appear as tight as previously claimed for all types of galaxies (e.g., Hu 2008; Graham 2008; Gültekin et al. 2009; Graham \& Li 2009). Significant outliers exist (e.g., Mathur et al. 2001; Grupe \& Mathur 2004; Watson et al. 2007), BHs are currently observed also in the centres of bulgeless galaxies (e.g., Ghosh et al. 2008; Satyapal et al. 2009; Araya salvo et al. 2012), and the AGN-starformation correlations disappear for all but highest luminosity AGNs (Shao et al. 2010; Grier et al. 2011).

Perhaps one of the most pressing reason to argue for supplementary routes of $\mathrm{BH}$ evolution is the prevalence of pseudobulges hosting BHs (e.g., Hu 2008; Gadotti \& Kauffmann 2009; Kormendy et al. 2011; Mathur et al. 2012; Orban de Xivry et al. 2011). "Pseudobulges" are structurally and dynamically different from "classical" bulges and ellipticals, which can also have very low specific angular momentum (e.g., Emsellem et al. 2011). They appear to have more "disky" profiles, they depart from the fundamental plane of elliptical galaxies, and are fainter at a fixed effective radius (e.g., Gadotti 2009).

At variance with elliptical galaxies and classical bulges, pseudobulges have been suggested to have formed through 
secular processes such as disk instabilities (Kormendy \& Kennicutt 2004). What we refer to here is bar instability, physically distinct to the clumpy instabilities that characterize the high- $z$, gas-rich disks (e.g., Daddi et al. 2010; Genzel et al. 2011).

Indeed, Shankar et al. (2012a) have recently shown that bulges mainly formed from bar-like disk instability in the Guo et al. (2011) model have much lower bulge-to-total (B/T) ratios and smaller sizes than classical bulges at fixed total stellar mass. Hopkins et al. (2010) have argued that the formation of pseudobulges depends on how gas-rich the merger is, and Fontanot et al. (2011) claim that it depends on the mass of the dark matter halo (see also discussions in Fanidakis et al. 2011; Yates et al. 2012). In particular, as detailed below, BHs residing in pseudobulges appear to be relatively undermassive compared to those in classical bulges of similar luminosity or velocity dispersion (e.g., $\mathrm{Hu}$ 2008).

Despite the strong observational efforts carried out over the years to characterize the $\mathrm{BH}$ population in later-type galaxies, theoretical models have mostly focused their attention on BHs in classical bulges. Here we investigate the formation of latetype bulges and their nuclear BHs, with emphasis on the class of galaxies broadly referred to as pseudobulges, in the framework of state-of-the-art hierarchical galaxy formation models that grow bulges and/or their central BHs via mergers and disk instabilities. In particular, we will show that mergers can produce a significant scatter in the $\mathrm{BH}$-galaxy mass scaling relations, in broad agreement with the observed properties of classical and pseudo bulges.

\section{Reference models}

The results discussed here are based on the online catalogues of the Bower et al. (2006, B06 hereafter) and the Guo et al. (2011, G11 hereafter) semi-analytic models ${ }^{1}$.

Both models follow the evolution of galaxies and their central BHs along the hierarchical evolution of their host dark matter haloes and subhaloes within the concordance $\Lambda$ CDM cosmology. To this purpose, they are both implemented on top of the same large, high-resolution cosmological $N$-body simulation MILLENNIUM I (Springel et al. 2005), making their comparison even more meaningful. The MILLENNIUM I simulation follows the evolution of $N=2160^{3}$ dark matter particles of mass $8.6 \times 10^{8} h^{-1} M_{\odot}$, within a comoving box of size $500 h^{-1} \mathrm{Mpc}$ on a side, from $z=127$ to the present, with cosmological parameters $\Omega_{\mathrm{m}}=0.25, \Omega_{\mathrm{b}}=0.045, h=0.73, \Omega_{\Lambda}=0.75, n=1$, and $\sigma_{8}=0.9$. The simulation can resolve haloes down to a mass of $\gtrsim 10^{10} M_{\odot} / h$, corresponding to BHs close to the seed mass used in the models $\left(\gtrsim 10^{4} M_{\odot}\right)$, thus, any conclusion drawn for BHs more massive than $\sim 10^{5} M_{\odot}$ is free of any resolution effect.

The primary channel to form bulges in both models is via mergers, during which bulges gain a fraction of the progenitor stars along with newly formed stars, both dependent on the strength and type of the merger.

Both models also substantially grow bulges via bar-like disk instabilities. Secular evolution is believed to form bulges via a bar that is capable of transferring mass from the disk to the central regions. At each timestep for each galactic system a stability criterion of the type

$V_{\max }<V_{\text {ref }}$,

${ }^{1}$ http://gavo.mpa-garching.mpg.de/MyMillennium3 is checked. Here $V_{\max }$ is the velocity at the maximum of the rotation curve, while $V_{\text {ref }} \propto \sqrt{G M_{\text {disk }} / R_{\text {disk }}}$ is proportional to the actual velocity of the disk via a factor close to unity.

When Eq. (1) is satisfied the models assume that the instability drives the formation of a bar from the disk into a central bulge (e.g., Combes et al. 1990; Cole et al. 2000). Despite the broad scheme of the disk instability is apparently similar in both models, there are significant differences. G11 treat disk instabilities as secular processes that act in self-gravitating disks near the instability boundary by transferring a portion of the stellar mass $\delta M_{\text {star }}$ from the disk to the bulge to keep the disk marginally stable with an exponential density profile.

B06 instead assume that the entire mass of the disk is transferred to the galaxy bulge when the disk goes unstable, with any gas present assumed to undergo a starburst and a fraction of it to feed the $\mathrm{BH}$. It is thus expected that disc instabilities play a more prominent role in the B06 model. B06 indeed find that in their model most of the BH growth occurs via disk instabilities rather than mergers. The disk instabilities considered by B06 may also qualitatively share some features with the instabilities mentioned in Sect. 1 characterizing high-z, gas-rich disks (e.g., Genzel et al. 2011), in the sense that in the B06 a significant fraction of gas can be fuelled to the centre even during disk instabilities. Nevertheless, the B06 are still considered and modelled as bar-like instabilities accompanied by a starburst (see discussion in Cole et al. 2000), thus still quantitatively different from the clumpy ones discussed in Sect. 1. Galaxy evolution models still lack a proper treatment of bulge formation (and any consequent $\mathrm{BH}$ feeding) via clump migration in gas rich, turbulent systems (see further discussions in Sect. 5 and in, e.g., Dekel et al. 2009).

Both models postulate that at each merger the central $\mathrm{BH}$ accretes mass proportionally to the available gas in the host galaxy (in the G11 model the accretion efficiency gets lower for smaller mass systems and for unequal mergers), as well as mass from other incoming BHs.

The most noticeable difference relevant to the present work is that, at variance with the G11 model, BHs in the B06 model also grow in mass during disk instabilities, proportionally to the fraction of gas available and undergoing a starburst at the moment of the disk instability. That some of the gas can be channelled into the very central regions during disk instabilities echoes some of the earlier models such as the so-called "bars within bars" scheme, proposed as a possible mechanism for fueling AGNs (e.g., Shlosman et al. 1989). For suitable conditions the gas inflow induced by the bar instability may produce a selfgravitating disk in a smaller region and a new small bar can develop, which induces further gas inflow.

In both models BHs also slightly grow in mass via hot accretion at late times (radio-mode feedback), but this is a minor channel of $\mathrm{BH}$ growth compared to cold gas accretion and $\mathrm{BH}-$ $\mathrm{BH}$ mergers. Anyway, including or neglecting such processes in the models is irrelevant to the present discussion.

Both models have been fine-tuned in their input parameters to reproduce the local scaling relations between $\mathrm{BH}$ mass and host galaxy stellar mass. However, the intrinsic scatter in the local relations is a genuine prediction of the models. The scatter reflects the relative diversity in the growth histories of central $\mathrm{BHs}$ hosted by galaxies of similar mass, induced by the intrinsic dispersion in the merger histories, and the different underlying physical assumptions (mergers and/or disk instability) to grow $\mathrm{BHs}$ in the models. Our discussion below will start from the comparison of the predicted scatter against the wealth of data now available on the $\mathrm{BH}$-galaxy scaling relations. 
Table 1. Galaxies hosting "pseudobulges" with known black hole masses.

\begin{tabular}{lccc}
\hline \hline Galaxy & $\log M_{\text {bh }}\left[M_{\odot}\right]$ & $\log M_{\text {bulge }}\left[M_{\odot}\right]$ & Reference \\
\hline NGC 1068 & 6.92 & 10.60 & H09 \\
NGC 3079 & 6.40 & 10.24 & H09 \\
NGC 3393 & 7.49 & 10.62 & H09 \\
Circinus & 6.04 & 9.62 & H09 \\
IC 2560 & 6.46 & 10.31 & H09 \\
MW & 6.61 & 10.11 & H09 \\
MW & 6.63 & 10.28 & K11 \\
Circinus & 6.08 & 10.14 & K11 \\
NGC 1068 & 6.93 & 11.20 & K11 \\
NGC 1300 & 7.85 & 10.14 & K11 \\
NGC 2748 & 7.67 & 9.80 & K11 \\
NGC 2787 & 7.61 & 9.80 & K11 \\
NGC 3227 & 7.18 & 9.97 & K11 \\
NGC 3384 & 7.26 & 10.52 & K11 \\
NGC 4736 & 6.82 & 10.42 & K11 \\
NGC 4826 & 6.13 & 10.30 & K11 \\
NGC 7582 & 7.74 & 10.29 & K11 \\
MS2254 & 6.60 & 10.25 & M11 \\
RX J1209 & 6.75 & 10.53 & M11 \\
RX J1117 & 7.33 & 10.49 & M11 \\
RX J2217 & 7.10 & 10.45 & M11 \\
RX J1702 & 7.34 & 10.53 & M11 \\
KUG1136 & 6.89 & 9.91 & O11 \\
Mrk 0042 & 6.22 & 9.91 & O11 \\
Mrk 0359 & 6.70 & 10.65 & O11 \\
Mrk 0382 & 6.59 & 10.26 & O11 \\
Mrk 0493 & 6.35 & 10.07 & O11 \\
Mrk 0766 & 7.14 & 9.61 & O11 \\
Mrk 0896 & 7.01 & 10.25 & O11 \\
Mrk 1044 & 7.20 & 10.03 & O11 \\
NGC 4748 & 6.95 & 10.07 & O11 \\
Mrk 0871 & 7.67 & 10.22 & W10 \\
Mrk 1126 & 6.90 & 9.80 & M01 \\
Circinus & 6.23 & 10.26 & S11 \\
IC 2560 & 6.64 & 10.94 & S11 \\
NGC 1068 & 6.93 & 11.33 & S11 \\
NGC 3079 & 6.40 & 11.02 & S11 \\
NGC 3368 & 6.88 & 10.74 & S11 \\
NGC 3489 & 6.78 & 10.07 & S11 \\
NGC 3998 & 8.38 & 10.36 & S11 \\
NGC 4258 & 7.58 & 10.98 & S11 \\
NGC 4594 & 8.76 & 11.23 & S11 \\
\hline
\end{tabular}

Notes. Details on the data listed in this table can be found in Sect. 3. Here H09, K11, M11, O11, S11, W10, and M01 refer to Hu (2012), Kormendy et al. (2011), Mathur et al. (2012), Orban de Xivry et al. (2011), Sani et al. (2011), Woo et al. (2010), and Mathur et al. (2001), respectively.

\section{Data}

In the following, we will compare model predictions with a collection of data taken from the Literature, listed in Table 1. These data are for active and inactive BHs the hosts of which have been mostly recognized as pseudobulges. Column 3 of Table 1 lists references for both $\mathrm{BH}$ and bulge masses. Where stellar masses were not directly available, we obtained them by converting $K$, $r$, or $V$ magnitudes using the mass-colour relations from Bell et al. (2003) with an average $g-r=0.5$, typical of these galaxies. All the data and the models have been converted to a common Chabrier (2003) Initial Mass Function. In Kormendy et al. (2011) BH masses have been measured using dynamical methods. In Mathur et al. (2012) and Orban de Xivry et al. (2011) widths of the $\mathrm{H} \beta$ emission lines in AGN spectra and the optical luminosity $L_{5100}$ have been used for BH mass estimates (see Mathur et al. 2011, for details. Orban de Xivry et al. do not list the BH masses in their paper; these have been kindly supplied by the authors; the $\mathrm{H} \beta$ width and $L_{5100}$ measurements are also reported in La Mura et al. 2007; Ohta et al. 2007; Sani et al. 2010; Orban de Xivry et al. 2011). Hu (2012) and Sani et al. (2011) report BH masses from multiple sources (please see their papers for details). Finally, we also add another two BH masses from Woo et al. (2010) and Mathur et al. (2001).

Different authors have used different techniques to estimate $\mathrm{BH}$ (and bulge) masses, and each technique has its own set of errors. The average error in $\mathrm{BH}$ masses for active and inactive galaxies is of the order of $\sim 0.3 \mathrm{dex}$ (see, e.g., Mathur et al. 2012). Of similar magnitude is the propagated error on stellar masses at fixed galaxy colour (see, e.g., Bell et al. 2003). In the following, for each galaxy we will use and show the multiple available $\mathrm{BH}$ and relative bulge mass estimates evaluated by each group. As it will be shown and discussed below, while some of the scatter in the data is undoubtedly induced by different measurement techniques, despite the different selections and methodologies, these data share a similar portion of the $M_{\mathrm{bh}}-M_{\text {bulge }}$ plane, mainly localized below the classical $M_{\mathrm{bh}}-M_{\text {bulge }}$ relation. The latter is not a new result and is discussed in the papers listed above. Here we simply put these data together for comparison with models.

Greene et al. (2008) and Jiang et al. (2011) have confirmed the results of the above mentioned works, extending them to lower BH masses. Greene et al. (2010) also report lower BH masses at fixed velocity dispersion for a (smaller) sample of local late-type galaxies with more accurate $\mathrm{BH}$ masses from maser kinematics. However, we do not report their results in Table 1 as only one galaxy in their sample with secure bulge masses is recognized as a pseudobulge. Also the indirect arguments put forward by Gadotti \& Kauffmann (2009) suggest pseudobulges have BHs less massive than their classical counterparts at fixed bulge mass or velocity dispersion.

\section{Results}

\subsection{A narrow and a broad distribution of Black Holes}

From now on we will simply refer to bulges grown mainly via bar-like disk instabilities as "pseudobulges", as opposed to "classical" bulges that have grown their mass mainly via mergers. Such a classification is rather arbitrary, though refined theoretical and observational work is now establishing that movement of gas and stars via bars to the centre could effectively contribute in building a young and kinematically cold stellar bulge component, that is, a disc-like/pseudo bulge (e.g., Gadotti 2011, and references therein). Moreover, it was recently pointed out by Shankar et al. (2012a) that galaxies of similar stellar mass that underwent a different merger history appear structurally different in the local Universe. In particular, they emphasized that in the G11 model those galaxies that had their bulges mostly grown via disk instability end up having significantly lower bulge-tototal ratios $B / T$ and lower half-mass radii at fixed stellar mass, in agreement with data (e.g., Gadotti 2009). Here we extend the differences emphasized by Shankar et al. (2012a) to BH masses and other properties.

The blue circles in the left panel of Fig. 1 are a random subsample of $1000 \mathrm{BHs}$ extracted from the B06 catalog, with the yellow, dashed line marking the median relation. A relatively narrow $M_{\mathrm{bh}}-M_{\text {bulge }}$ relation is predicted in fair agreement with what empirically calibrated in the local Universe (the grey band is the $M_{\mathrm{bh}}-M_{\text {bulge }}$ fit for classical bulges by Sani et al. 2011, with 


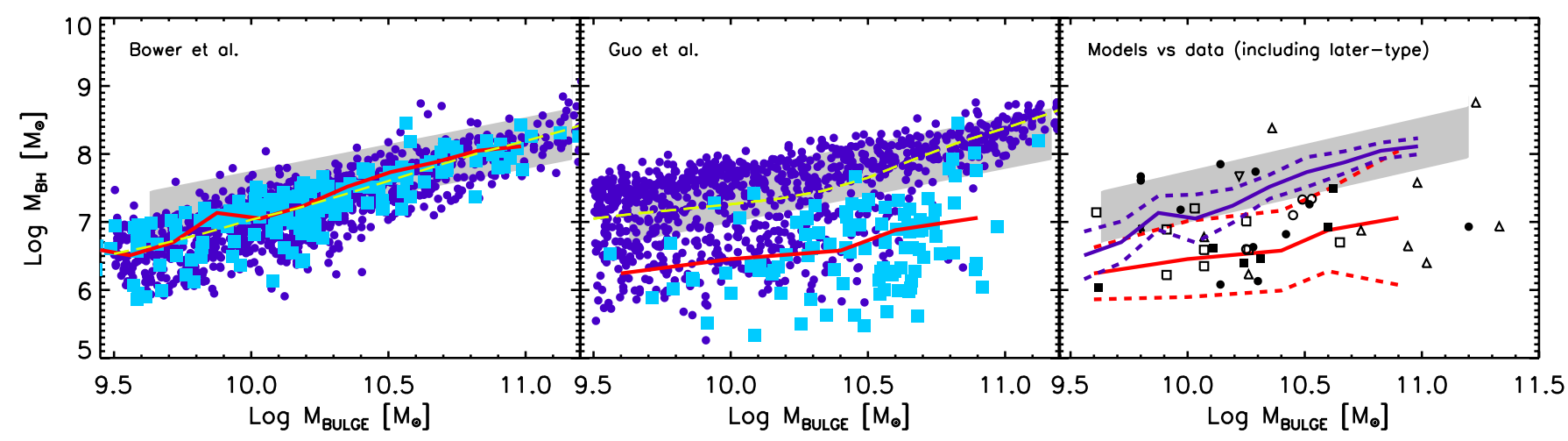

Fig. 1. Left panel: the dark blue circles show the distribution of black holes for a sample of 1000 black holes randomly selected from the Bower et al. (2006) catalogue. The cyan squares are a random sample of 100 black holes with their hosts' bulges mainly grown via secular processes ("pseudobulges"). The long-dashed yellow and red black lines are the median $M_{\mathrm{bh}}-M_{\mathrm{bulge}}$ relations competing to the former and the latter samples, respectively; the grey stripe indicates the local empirical $M_{\mathrm{bh}}-M_{\text {bulge }}$ relation for classical bulges from Sani et al. (2011). Middle panel: same pattern as the left panel but with both samples extracted from the Guo et al. (2011) catalog, with the long-dashed yellow and red solid lines being the respective median $M_{\mathrm{bh}}-M_{\text {bulge }}$ relations for the two samples. Right panel: comparison between predicted the distributions of pseudobulges of Bower et al. (2006) and Guo et al. (2011; blue and red lines, respectively), with their 1- $\sigma$ uncertainties (dashed lines) compared to data (Table 1) from $\mathrm{Hu}$ (2012, filled squares), Kormendy et al. (2011, filled circles), Mathur et al. (2012, open circles), Orban de Xivry et al. (2011, open squares), Woo et al. (2010, upside down triangle), and Mathur et al. (2001, filled star). Also shown for completeness data on pseudobulges by Sani et al. (2011, open triangles). The average error in BH masses for active and inactive galaxies is of the order of $\sim 0.3$ dex. Of similar magnitude is the propagated error on stellar masses at fixed galaxy colour.

its intrinsic scatter; similar results were found by, e.g., Häring \& Rix 2004, though the latter are mainly based on dynamical masses). The cyan solid squares represent 100 randomly extracted BHs hosted by pseudobulges, with the red, long-dashed line marking the median relation. These galaxies were initially selected from the catalogs as "potential" pseudobulges for having lower $B / T$ (usually set to be $B / T \lesssim 0.5$ ) at fixed bulge stellar mass. By analyzing their merger trees, following Shankar et al. (2012a) we then selected and defined as pseudo (classical) bulges those galaxies that had grown more (less) than $50 \%$ of their bulge mass via disk-instability. We repeated the exercise of randomly extracting 100 galaxies with such characteristics several times finding similar results. Given that BHs even in disk-instability mode in the B06 model grow proportionally to bulges, it is not unexpected that they will end up following similar scaling relations to the $\mathrm{BHs}$ in classical bulges.

The middle panel of Fig. 1 shows the predictions of the G11 model. The predicted $M_{\mathrm{bh}}-M_{\text {bulge }}$ relation (blue circles), with the median shown with a yellow, long-dashed line, is still broadly consistent with local data, though the predicted scatter at fixed stellar mass is much larger in this model. The cyan squares are a subsample of 100 randomly selected $\mathrm{BHs}$ that have grown more than $50 \%$ of their bulge mass via bar-like disk instability. Galaxies of the latter type were again initially selected as having lower $B / T$ at fixed bulge mass. It is clear that extracting merger histories for the millions of $\mathrm{BHs}$ in the online catalogues becomes prohibitive. Nevertheless, for the G11 model we compared our randomly selected pseudobulge sample with one directly extracted from the Shankar et al. (2012a) catalog. The latter is a rendition of the G11 model with additional information on bulge sizes, where the ratio between size growth via merger and bar instability was self-consistently followed for each galaxy in the model (see further details in Shankar et al. 2012a). From such a catalog we randomly extracted 100 galaxies with bulge sizes mostly grown via bar instabilities finding very similar results compared to our random samples.

In the right panel we compare model predictions with a collection of data taken from the Literature, listed in Table 1 and discussed in Sect. 3. We stress that these data are for active and inactive BHs the hosts of which have been recognized as pseudobulges. These data show that despite the different selections and methodologies, they share a similar portion of the $M_{\mathrm{bh}^{-}}$ $M_{\text {bulge }}$ plane. The most striking feature shown by this collection of data is that at fixed bulge mass, most of the objects are mainly localized below the classical $M_{\mathrm{bh}}-M_{\text {bulge }}$ relation, characterized by the grey band, by up to a factor of $\lesssim 100$ and over nearly two orders of magnitude in bulge stellar mass. The blue and red lines are the predictions for pseudobulges of the B06 and G11 models, respectively, with the solid and dashed lines marking their respective mean and $1-\sigma$ uncertainties (computed from the 16th and 84th percentile of the $\log M_{\mathrm{bh}}$ distributions competing to each bin of bulge stellar mass).

We find that the B06 model predicts a rather tight $M_{\mathrm{bh}}-M_{\text {bulge }}$ relation for all galaxies with normalization consistent with that of classical bulges. Thus the model can only marginally account for BHs in later type galaxies that observationally mostly lie well below the classical $M_{\mathrm{bh}}-M_{\text {bulge }}$ relation. The G11 model performs better in this respect. Although a significant fraction of the local BH population clusters around the classical $M_{\mathrm{bh}}-M_{\text {bulge }}$ relation, a non-negligible portion extends to very low mass BHs

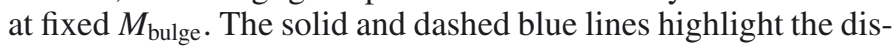
tribution of galaxies in the G11 model that have grown most of their bulge mass via disk instabilities. More precisely, we find that moving, at fixed $M_{\text {bulge }}$, from the lowest mass BHs to the highest ones, the fraction of mass assembled via mergers gradually increases. The merger model therefore does not predict a bimodality of BHs in classical and pseudo bulges, but rather a continuous trend.

A more quantitative comparison between models and data is provided in Fig. 2 which shows $\mathrm{BH}$ mass distributions for a fixed bin of bulge stellar mass. Given that we are here mainly interested in the comparison among predicted and measured scatters in $\mathrm{BH}$ mass at fixed bulge mass, we simply normalize each histogram to have the same peak at 1 . Distributions for galaxies with bulge masses $10<\log M_{\text {bulge }}<10.5$ and $10.5<\log M_{\text {bulge }}<11$ are plotted in the left and right panels, respectively. The red and blue lines are the predictions for the G11 and B06 models, respectively, separated for classical and 
F. Shankar et al.: Black holes in pseudobulges: demography and models

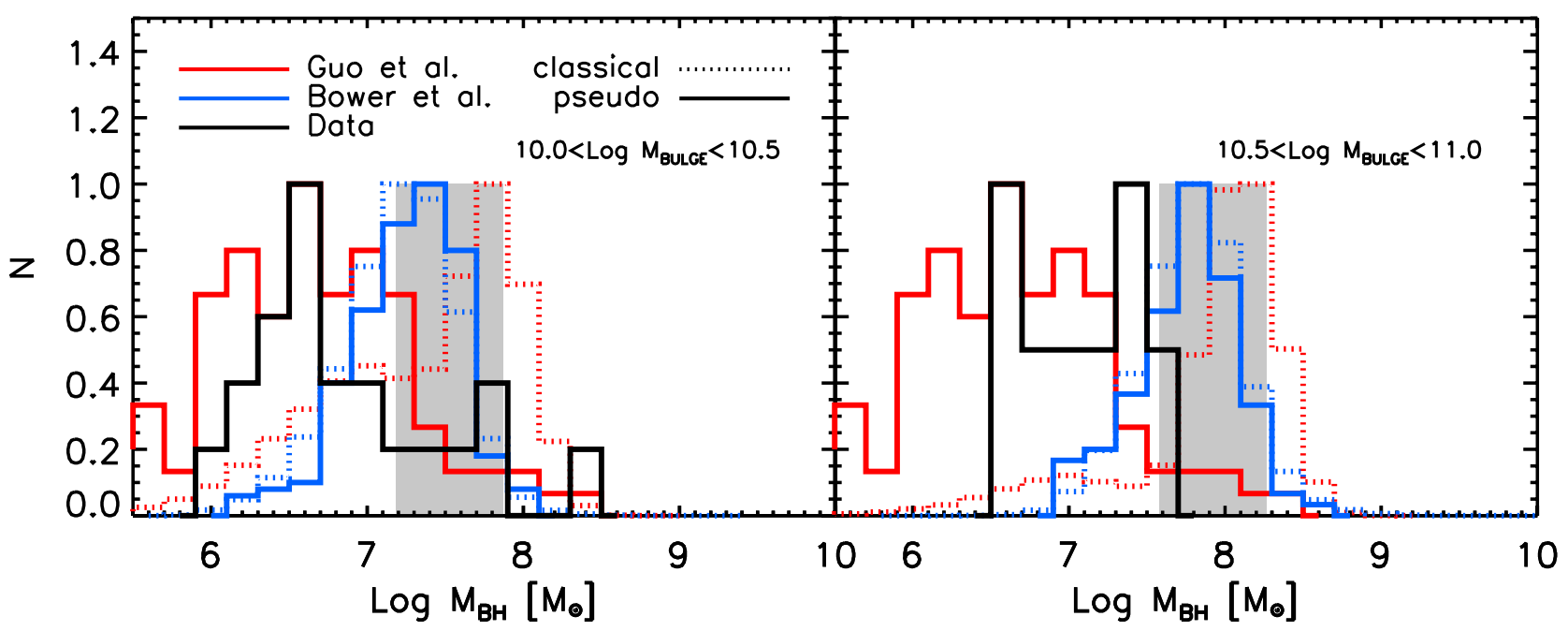

Fig. 2. Black hole mass distributions for galaxies with bulge masses $10<\log M_{\text {bulge }}<10.5$ (left panel) and $10.5<\log M_{\text {bulge }}<11$ (right panel) predicted by the Guo et al. (2011) and Bower et al. (2006) models (red and blue lines, respectively), and for classical and pseudo bulges (dotted and solid lines, respectively). The solid, black lines in both panels are the data collected in Table 1. The grey stripes are the black hole mass intervals (median plus scatter) inferred from the Sani et al. $M_{\mathrm{bh}}-M_{\mathrm{bulge}}$ relation for the corresponding bulge masses.

pseudo bulges (dotted and solid lines, respectively). The solid, black lines in both panels are the data collected in Table 1, while the grey stripes are the black hole mass intervals (median plus scatter) inferred from the Sani et al. (2011) $M_{\mathrm{bh}}-M_{\text {bulge }}$ relation for the corresponding mean bulge masses.

For each $\log M_{\mathrm{bh}}$ distribution we then compute the median value, and the 16th and 84th percentile which define the $\pm 1 \sigma$ errors on $\log M_{\mathrm{bh}}$. We find that, at least above $\log M_{\text {bulge }} \gtrsim 11$ both models predict a narrower distributions for classical bulges, with an intrinsic $\sigma\left(\log M_{\mathrm{bh}}\right) \sim 0.3-0.4$ dex, in good agreement with the empirical one of 0.38 dex (grey band). However, while the B06 model continues to predict a similarly narrow distribution for pseudobulges, the G11 model produces much broader distributions for pseudobulges, with an intrinsic scatter of $\sigma\left(\log M_{\mathrm{bh}}\right) \sim 0.6 \mathrm{dex}$, a factor of about two higher than for the B06 model and in better agreement with the data (cf. solid, red lines, the G11 model/pseudo, with the black, solid histograms, the data). At lower bulge masses (left panel), both models envision similar BH mass distributions and similar differences, with the G11 model better matching observations.

In the $\mathrm{G} 11$ model seed $\mathrm{BHs}$ ( set to $10^{3} M_{\odot}$ ) grow via mergers by orders of magnitude to the level of $\gtrsim 10^{5}-10^{7} M_{\odot}$, comparable to the $\mathrm{BH}$ masses measured in late-type galaxies. At later times, when mergers become rarer, $\mathrm{BH}$ growth is blocked while host galaxies continue to grow via in-situ star formation and bulges grow via disk instabilities. Thus the predicted $M_{\mathrm{bh}}-M_{\text {bulge }}$ relation in the G11 model will inevitably show a rather large scatter at fixed bulge mass.

\subsection{Pseudobulges and classical bulges: evolution}

In Fig. 3 we show several mean properties for the subsamples of pseudobulges of the B06 and G11 models discussed earlier (solid black and blues lines, respectively), compared with the mean properties of subsamples of classical bulges (red longdashed and dot-dashed lines, respectively). For each object we trace back its more massive progenitor in the merger tree and record its properties. Shankar et al. (2012a) found that the sizes of pseudobulges are predicted to be a factor of a few lower than those of classical bulges at fixed stellar mass. Figure 2 extends their study to other properties.

\subsection{1. $B / T$ versus $z$}

We find that the bulge-to-total $B / T$ ratios (upper middle) of both galaxy populations increase rapidly at very early epochs. However, while classical bulges continue to significantly grow and turn into ellipticals $(B / T \sim 1)$ at late times, the $B / T$ of pseudobulges quickly flatten out to values $B / T \lesssim 0.6$ after a few Gyrs, as a consequence of their significantly different growth patterns $^{2}$.

\subsubsection{SFR versus $z$}

Pseudobulges in both models reside in lower mass haloes at $z \lesssim 2$ (lower left), which contributes in explaining the different merger histories; it also induces less powerful mergers, thus less $\mathrm{BH}$ growth at all times. We find in fact that pseudobulges have grown $>70 \%$ of their final stellar mass via disk instabilities (see also discussion in B06), while classical bulges of the same mass tend to grow mostly via late dry minor mergers (see details in Shankar et al. 2012a, and references therein). This is also evident from their different cosmological star formation rate (SFR; lower middle; information on the SFR are only available for the G11 catalogue) and specific SFR (SSFR; lower right). The SFR in pseudobulges peaks at later times and their SSFR is larger by a factor of a few at $z \lesssim 2.5$ with respect to classical bulges that become progressively dead with time though still significantly increasing in stellar mass (via dry mergers).

\subsection{3. $\mathrm{BH}$ mass versus $z$}

The characteristic BH mass (upper right) in pseudobulges for the G11 model (solid blue) is a factor of several lower ( $\gtrsim 4-10$ depending on redshift) than those in classical bulges (red dotdashed) since early times, at variance with the bulge masses that differ by only a factor $\$ 2$ (upper left). The B06 model instead predicts that both $\mathrm{BH}$ and bulge masses of pseudo (solid black) and classical (red long dashed) bulges are similar because they

\footnotetext{
2 The apparent slight decrease in the $B / T$ for pseudobulges in the B06 model is simply an artifact of the averaging, the large intrinsic scatter, and the choice of galaxy subset, but not driven by any evident physical process.
} 

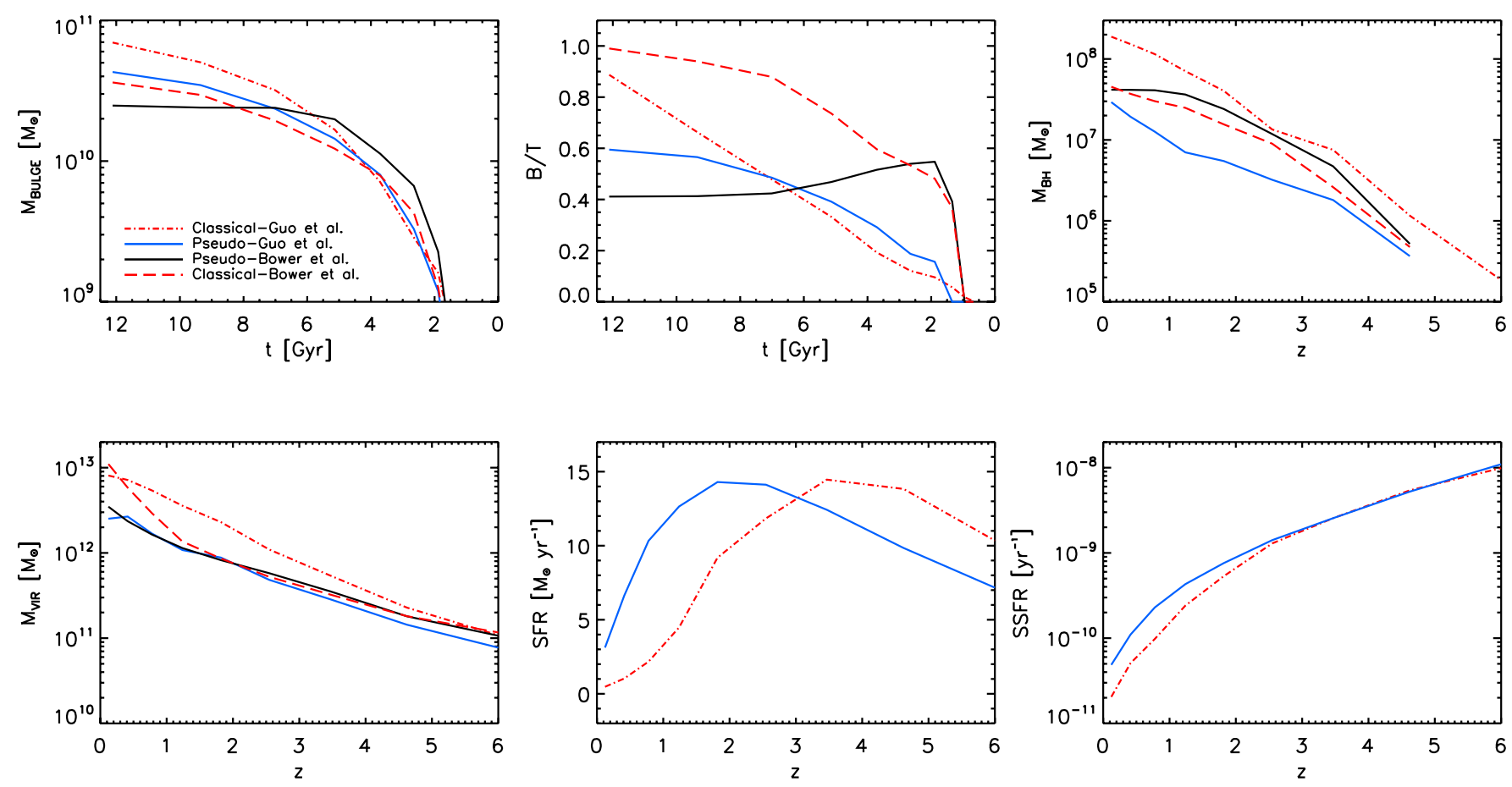

Fig. 3. Comparison among the median properties of random subsamples of classical and pseudobulges from the Guo et al. (2011) model (red, dot-dashed and blue, solid lines, respectively) and Bower et al. (2006) model (red, long-dashed and black, solid lines, respectively). Upper panels: bulge mass (left), bulge-to-total $B / T$ ratio (middle), and black hole mass (right) as a function of cosmic time or redshift. Lower panels: subhalo virial host mass (left), star-formation rate (middle), and specific star-formation rate (right) as a function of redshift. Overall, pseudobulges appear to have prolonged star-formation rates, significantly lower $B / T$, and in models with only mergers as fuelling mechanism, lower mass black holes, in agreement with observational data.

always co-evolve. At late times pseudobulges continue forming stars in their disks, undergoing disk instabilities, and growing their bulges. However BHs in the G11 model suffer severe starving due to less numerous and less powerful merger events, thus inducing further departure from the classical $M_{\mathrm{bh}}-M_{\mathrm{bulge}}$ relation $^{3}$. We'd like to conclude stressing that what discussed here are possibly extreme BHs in pseudo and classical bulges, but the models also predict significant fractions of BHs with intermediate properties.

\subsection{Late-type bulges: BH demography revisited}

Having compared theoretical and observational relations of BHs in classical and pseudo-bulges, we here move on to discussing the full demography of the predicted and observed BH populations via the $\mathrm{BH}$ mass functions. While this type of comparison was pursued several times in the Literature (e.g., Lapi et al. 2006; Marulli et al. 2008), it was never performed before taking care of differentiating classical and pseudo bulges.

To this purpose, we first recompute an empirical estimate of the local BH mass function (BHMF). A common way to obtain an estimate of the BHMF is via convolution of the velocity dispersion function (VDF) of local galaxies, with the $M_{\mathrm{bh}}-\sigma$ relation (the convolution is between the VDF and a Gaussian

\footnotetext{
3 We note that each subset of mock galaxies was mostly chosen on the basis of being enough massive to host a super-massive $\mathrm{BH}$, and on the relative fractional growth via mergers and disc instabilities. We thus expect by construction the pseudo and classical bulge subsets within each model to yield similar stellar masses at $z=0$ (at the factor of $\lesssim 2$ level). However, we do not expect the classical and/or pseudobulge subsamples between the two model to necessarily yield the same bulge, $\mathrm{BH}$, or virial mass at $z=0$.
}

with dispersion equal to the intrinsic scatter in the $M_{\mathrm{bh}}-\sigma$ relation). To this purpose, we use the latest determination of the Hubble type-dependent VDF from Bernardi et al. (2010), coupled with a revised $M_{\mathrm{bh}}-\sigma$ relation by Shankar et al. (in prep.) obtained from an updated sample of Ferrarese \& Ford (2005), i.e., $\log M_{\mathrm{bh}}=(4.57 \pm 0.47) \log \sigma+8.26 \pm 0.08$ with intrinsic scatter $\eta=0.17 \pm 0.08$ (for early-type galaxies), and $\log M_{\mathrm{bh}}=(4.99 \pm 0.38) \log \sigma+8.18 \pm 0.06$ with intrinsic scatter $\eta=0.17 \pm 0.06$ for later-type galaxies (though similar conclusions are derived adopting other estimates of the relation). The black, dot-dashed line in Fig. 4 is the result of the convolution including all Hubble types, with the dotted lines marking the 1- $\sigma$ error bars derived from Monte Carlo simulations that include statistical uncertainties on the VDFs and scaling relations (including uncertainties in the intrinsic scatter). The estimated $\mathrm{BH}$ mass function is in good agreement with previous estimates (e.g., Shankar et al. 2009, grey band).

However, Fisher \& Drory (2011) recently suggested that pseudobulges may be the dominant class of bulges at low masses. Moreover, as discussed above, pseudobulges host significantly lower mass BHs than expected from the $M_{\mathrm{bh}}-\sigma$ relation. The long-dashed, cyan line in Fig. 4 shows the local BH mass function obtained by assuming that all bulges in $\mathrm{Sb}$ galaxies are actually pseudobulges and thus follow the $M_{\mathrm{bh}}-\sigma$ relation by $\mathrm{Hu}$ (2008). (We do not claim that there is a separate $M_{\mathrm{bh}}-\sigma$ relation for pseudobulges; the $\mathrm{Hu} 2008$, relation is used here just for illustration.) The solid, cyan line is the extreme estimate in which all bulges in $\mathrm{Sb}$ and $\mathrm{Sa}$ galaxies are pseudobulges. This exercise shows that accounting for pseudobulges can easily decrease the $\mathrm{BH}$ mass density by $\sim 15 \%$, when correcting only $\mathrm{Sb}$, up to $\sim 35 \%$ in the extreme case that all $\mathrm{Sb} / \mathrm{Sa}$ galaxies are actually pseudobulges, while BH number densities decrease by a factor 
F. Shankar et al.: Black holes in pseudobulges: demography and models

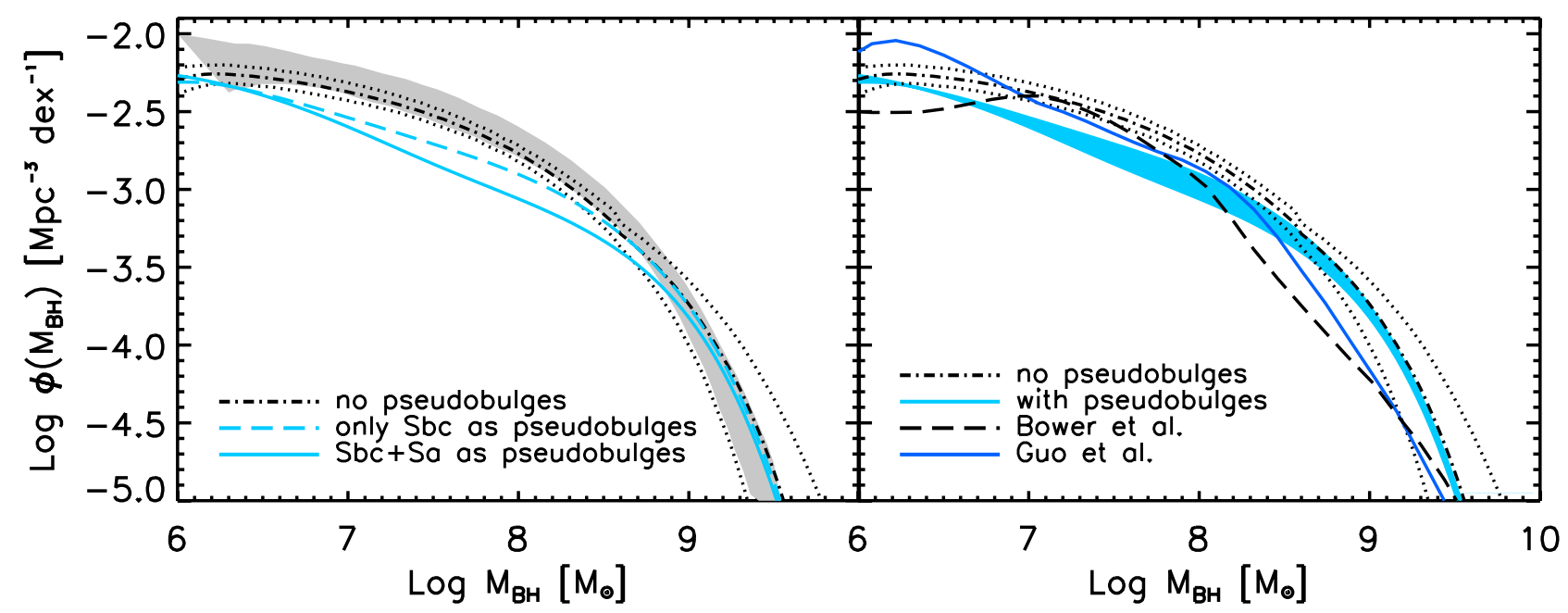

Fig. 4. Left panel: estimate of the local black hole mass function derived by convolution of the galaxy velocity dispersion function and the $M_{\mathrm{bh}}-\sigma$ relation considering all bulges as classical (dot-dashed and dotted lines), compared to the collection of estimates from Shankar et al. (2009, grey band). The cyan solid line is the estimate of the local black hole mass function assuming that all Sbc+Sa galaxies follow the Hu (2012) $M_{\mathrm{bh}}-\sigma$ relation proper of pseudobulges. The cyan dot-dashed line is the local black hole mass function obtained assuming that only Sbc are pseudobulges. Right panel: predicted local black hole mass function from the Bower et al. (2006) and Guo et al. (2011) models (black long-dashed and blue solid lines, respectively) compared with the local mass function not corrected (dot-dashed and dotted lines) and corrected for pseudobulges (cyan area, combination of the dot-dashed and solid lines in the left panel).

of $\sim 2-3$ below $\lesssim 10^{8} M_{\odot}$ (these results do not depend much on the assumed scatter in the Hu 2008, relation).

The right panel of Fig. 4 compares the local $\mathrm{BH}$ mass function corrected for pseudobulges (cyan area, obtained by combining the cyan long-dashed and solid lines in the left panel), with the global BH mass function at $z=0$ predicted by the B06 and G11 models (long-dashed, black and solid, blues lines, respectively). It is interesting to note that both models still predict too many classical (i.e., more massive at fixed $M_{\text {bulge }}$ ) BHs in the local Universe, thus tending to overpredict the number of low mass $\mathrm{BHs}$, in the range $10^{7}$ and $10^{8} M_{\odot}$, with respect to the empirical $\mathrm{BH}$ mass function corrected for pseudobulges (below $10^{7} M_{\odot}$, the number density continues to be overpredicted by G11, but it is underpredicted by B06).

From this rather straightforward experiment we thus conclude that if $\mathrm{BHs}$ in pseudobulges really are the most common type of BHs in the local Universe, then models still need to properly account for such a large population (see also Fontanot et al. 2011; Shankar et al. 2012a). Recent estimates of the local BH mass function (e.g., Vika et al. 2009) claim even lower number densities of low mass BHs that would further exacerbate the tension with model predictions. The shape of the local $\mathrm{BH}$ mass function at low masses is essential to properly constrain the total amount of sub-Eddington accretion that characterized the cosmic evolution of BHs (Shankar et al. 2012b).

\section{Discussion}

The comparison in Fig. 1 suggests that a tight link between bar instabilities and $\mathrm{BH}$ growth at all times and in all conditions may be disfavored. More specifically, we have explored the outputs of the B06 model that couples BH growth to mergers and disk instabilities with both fuelling modes tuned to match the median local scaling relation between BHs and bulges masses in earlytype galaxies. We have then compared the model outputs against a (recent) collection of data on $\mathrm{BH}$ masses in local active and inactive galaxies of late-type morphological type, mostly identified as pseudobulges, that tend to be outliers with respect to the scaling relations of BHs in earlier-type, classical bulges. The model struggles to fully reproduce the latter selection of data. In other words, the intrinsic scatter predicted by the B06 model induced by differences in galaxy-to-galaxy evolutionary paths is not sufficient by itself to match the recent data on BHs in later type galaxies/pseudobulges.

The G11 model instead, triggering BHs only in mergers, allows for significant, late bulge growth via bar instabilities with no parallel fuelling in the central $\mathrm{BH}$, thus naturally creating a larger scatter in the $\mathrm{BH}$-bulge mass relations. Our results thus suggest that models in which late time disk instabilities, mainly characterized by the formation of bars, are not closely related to $\mathrm{BH}$ growth, tend to generate a larger scatter in the $M_{\mathrm{bh}}-M_{\text {bulge }}$ relation in better agreement with the broad distribution of BHs we observe today. This finding confirms prior expectations (see, e.g., Kormendy et al. 2011; Mathur et al. 2012, and references therein).

We note here that several other galaxy/AGN evolutionary models based on $\mathrm{BH}$ fuelling in bulges with no bar instabilities (e.g., Granato et al. 2004; Cirasuolo et al. 2005; Shankar et al. 2006) have also suggested additional possible sources of the observed large scatter observed in $\mathrm{BH}$ scaling relations. A break in $\mathrm{BH}$ scaling relations at low masses induced by inefficiency of quasar compared to supernova feedback, responsible for shaping the galaxy stellar mass function (e.g., Shankar et al. 2006, and references therein), and different normalizations of the $M_{\mathrm{bh}^{-}}$ $M_{\text {bulge }}$ relation at different virialization epochs of the host haloes, inevitably increase the predicted distribution of $\mathrm{BH}$ masses at fixed bulge mass.

Our results, however, do not imply that $\mathrm{BH}$ fuelling triggered by disk instabilities is never at work. Within the highly turbulent interstellar medium of the protogalactic disks at high redshifts mentioned in Sect. 1 (e.g., Genzel et al. 2011), large clumps can form and migrate towards the centre via dynamical friction. At variance with bars that can build up bulges gradually on very long timescales, the latter type of instabilities are rapid and violent processes. Moreover, the clumpy disk instabilities may potentially form central spheroids structurally more 
similar to early-type bulges, as displayed by some recent numerical simulations (e.g., Bournaud et al. 2011a; but see also Inoue \& Satoh 2011). The clumpy inflows within high- $z$ disks have been recently recognized to also play a non-negligible role in feeding the central BHs (Schawinski et al. 2011; Bournaud et al. 2011b), possibly under the effect of continuous cold flow accretion (Di Matteo et al. 2012). In this framework, the turbulent disks at high redshifts seem to share similar effects to mergers, forming classical bulges and proportionally growing central BHs (e.g., Bournaud et al. 2011b, and references therein).

Our results may equivalently reflect on a still not fully appropriate treatment of $\mathrm{BH}$ fuelling during bar-like disk instability events. The way BH feeding follows late bar instabilities may in fact be a non-trivial function of other parameters such as gas fractions, environment, etc. that may further contribute to produce the local scatter we observe.

Our understanding of BH feeding is in fact still fuzzy, especially at lower redshifts when gas reservoirs in galaxies become poorer. Peeples \& Martini (2006) raised some doubts about a strong link between bars and AGN activity. They investigated the correlation between bar strength and circumnuclear dust morphology for 75 galaxies finding that in many cases strongly barred galaxies showed structures terminating in a circumnuclear starburst ring at $\sim 10 \mathrm{pc}$ away from the galactic nucleus. On the other hand, many Narrow Line Seyfert 1 galaxies appear as barred systems and their AGNs are believed to be fuelled via bars (e.g., Crenshaw et al. 2003; Orban de Xivry et al. 2011).

Coelho \& Gadotti (2011) recently claimed evidence for double the fraction of AGNs in barred galaxies as compared to unbarred galaxies, at least for low-mass bulges. Oh et al. (2012) have recently analyzed a large sample of barred and unbarred galaxies extracted from the Sloan Digital Sky Survey concluding that the correlation between bars and AGN activity (and SFR) is far from trivial and also difficult to establish due to secondary correlations between AGNs, galaxy morphology, etc. They however point out that some connection between AGN activity and bars seems to be present, at least in the local Universe. In particular, they claim that galaxies having longer bars appear to have more AGNs than their counterparts at fixed stellar mass and morphology.

Indirect evidence for $\mathrm{BH}$ growth via bars may also come from higher redshifts. Cisternas et al. (2011) find the BH to total host stellar mass ratio to be constant since redshift $z=0.9$. Given that their sample is mainly composed by galaxies with disks, they claim that secular processes might have fuelled both a central bulge and correlated $\mathrm{BH}$ in time thus preserving their correlation down to $z=0$. More generally, other additional sources of BH fuelling at later times are also probably at work, such as winds from stars (e.g., Ciotti \& Ostriker 2001; Kauffmann \& Heckman 2009; Cen 2012, and references therein).

Overall, evidence for some AGN activity in barred/pseudobulged systems may be present. However, there is also mounting evidence that these systems lie mostly below the relations characterizing classical bulges, for both active and inactive galaxies (e.g., Graham et al. 2011). Beifiori et al. (2012) have recently revisited a number of BH scaling relations in a revised sample of local galaxies with $\mathrm{BHs}$, and claimed, in line with Kormendy et al. (2011), that pseudobulges have little or no correlation between $\mathrm{BH}$ mass and velocity dispersion. Thus, it is not at all certain that BHs in non-classical bulges should end up obeying similar scaling laws. Orban de Xivry et al. (2011), for example, suggest that Narrow Line Seyfert 1 may not be in any special phase of their evolution, but simply are BHs growing slowly due to their specific duty cycle and spin, mainly driven by secular processes in their pseudobulge hosts.

As discussed in Sect. 4.3, Fisher \& Drory (2011) recently suggested that pseudobulges may be the dominant class of bulges at low masses. Thus, the number of outliers in the BH-galaxy classical relations could be very high, impacting the demography of local BHs by severely reducing the expected number density of low mass BHs in the local Universe (similar thoughts were also put forward by Gadotti \& Kauffmann 2009; Greene et al. 2010). This in turn could have a non-negligible repercussion on accretion and merging models that try to tune parameters such as the radiative efficiency and Eddington ratio distributions by matching the full shape of the local $\mathrm{BH}$ mass function (e.g., Shankar 2009; Marulli et al. 2008; Shankar et al. 2012b).

Acknowledgements. F.S. acknowledges support from a Marie Curie Fellowship. We thank the referee for a thoughtful report that helped to significantly improve the presentation of the paper. We thank N. Fanidakis, L. Ferrarese, F. Fontanot, P. Nair, R. Yates, and G. Zamorani for interesting discussions, and E. Sani and G. Orban de Xivry for providing us with their data.

\section{References}

Araya salvo, C., Mathur, S., Fiore, F., \& Ferrarese, L. 2012, ApJ, submitted Barnes, J. E. 1992, ApJ, 393, 484

Beifiori, A., Courteau, S., Corsini, E. M., \& Zhu, Y. 2012, MNRAS, 419, 2497 Bell, E. F., McIntosh, D. H., Katz, N., \& Weinberg, M. D. 2003, ApJ, 585, L117 Bernardi, M., Shankar, F., Hyde, J. B., et al. 2010, MNRAS, 404, 2087

Bournaud, F., Chapon, D., Teyssier, R., et al. 2011a, ApJ, 730, 4

Bournaud, F., Dekel, A., Teyssier, R., et al. 2011b, ApJ, 741, L33

Bower, R. G., Benson, A. J., Malbon, R., et al. 2006, MNRAS, 370, 645

Cen, R. 2012, ApJ, submitted [arXiv: 1102.0262]

Chabrier, G. 2003, ApJ, 586, L133

Ciotti, L., \& Ostriker, J. P. 2001, ApJ, 551, 131

Cirasuolo, M., Shankar, F., Granato, G. L., De Zotti, G., \& Danese, L. 2005, ApJ, 629,816

Cisternas, M., Jahnke, K., Bongiorno, A., et al. 2011, ApJ, 741, L11

Coelho, P., \& Gadotti, D. A. 2011, ApJ, 743, L13

Cole, S., Lacey, C. G., Baugh, C. M., \& Frenk, C. S. 2000, MNRAS, 319, 168 Combes, F., Debbasch, F., Friedli, D., \& Pfenniger, D. 1990, A\&A, 233, 82 Crenshaw, D. M., Kraemer, S. B., \& Gabel, J. R. 2003, AJ, 126, 1690

Daddi, E., Bournaud, F., Walter, F., et al. 2010, ApJ, 713, 686

Dekel, A., Birnboim, Y., Engel, G., et al. 2009, Nature, 457, 451

Di Matteo, T., Khandai, N., DeGraf, C., et al. 2012, ApJ, 745, L29

Emsellem, E., Cappellari, M., Krajnović, D., et al. 2011, MNRAS, 414, 888

Fanidakis, N., Baugh, C. M., Benson, A. J., et al. 2011, MNRAS, 410, 53

Ferrarese, L., \& Ford, H. 2005, Space Sci. Rev., 116, 523

Ferrarese, L., \& Merritt, D. 2000, ApJ, 539, L9

Fisher, D. B., \& Drory, N. 2011, ApJ, 733, L47

Fontanot, F., De Lucia, G., Wilman, D., \& Monaco, P. 2011, MNRAS, 416, 409

Gadotti, D. A. 2009, MNRAS, 393, 1531

Gadotti, D. A. 2011, MNRAS, 415, 3308

Gadotti, D. A., \& Kauffmann, G. 2009, MNRAS, 399, 621

Genzel, R., Newman, S., Jones, T., et al. 2011, ApJ, 733, 101

Ghosh, H., Mathur, S., Fiore, F., \& Ferrarese, L. 2008, ApJ, 687, 216

Graham, A. W. 2008, ApJ, 680, 143

Graham, A. W., \& Li, I.-H. 2009, ApJ, 698, 812

Graham, A. W., Onken, C. A., Athanassoula, E., \& Combes, F. 2011, MNRAS, 412,2211

Granato, G. L., De Zotti, G., Silva, L., Bressan, A., \& Danese, L. 2004, ApJ, 600,580

Granato, G. L., Silva, L., Lapi, A., et al. 2006, MNRAS, 368, L72

Greene, J. E., Ho, L. C., \& Barth, A. J. 2008, ApJ, 688, 159

Greene, J. E., Peng, C. Y., Kim, M., et al. 2010, ApJ, 721, 26

Grier, C. J., Mathur, S., Ghosh, H., \& Ferrarese, L. 2011, ApJ, 731, 60

Grupe, D., \& Mathur, S. 2004, ApJ, 606, L41

Gültekin, K., Richstone, D. O., Gebhardt, K., et al. 2009, ApJ, 698, 198

Guo, Q., White, S., Boylan-Kolchin, M., et al. 2011, MNRAS, 413, 101

Häring, N., \& Rix, H.-W. 2004, ApJ, 604, L89

Hopkins, P. F., Bundy, K., Croton, D., et al. 2010, ApJ, 715, 202

Hu, J. 2008, MNRAS, 386, 2242

Hu, J. 2012, MNRAS, submitted [arXiv:0908.2028]

Jiang, Y.-F., Greene, J. E., \& Ho, L. C. 2011, ApJ, 737, L45 
F. Shankar et al.: Black holes in pseudobulges: demography and models

Kauffmann, G., \& Heckman, T. M. 2009, MNRAS, 397, 135

Kormendy, J., \& Bender, R. 2012, ApJ, 198, 2

Kormendy, J., \& Kennicutt, Jr. R. C. 2004, ARA\&A, 42, 603

Kormendy, J., Bender, R., \& Cornell, M. E. 2011, Nature, 469, 374

La Mura, G., Popović, L. Č., Ciroi, S., Rafanelli, P., \& Ilić, D. 2007, ApJ, 671, 104

Lapi, A., Shankar, F., Mao, J., et al. 2006, ApJ, 650, 42

Lapi, A., Gonzalez-Nuevo, J., Fan, L., et al. 2011, ApJ, 742, 24

Magorrian, J., Tremaine, S., Richstone, D., et al. 1998, AJ, 115, 2285

Marulli, F., Bonoli, S., Branchini, E., Moscardini, L., \& Springel, V. 2008, MNRAS, 385,1846

Mathur, S., Kuraszkiewicz, J., \& Czerny, B. 2001, NewAR, 6, 321

Mathur, S., Fields, D., Peterson, B. M., \& Grupe, D. 2012, ApJ, submitted [arXiv: 1102.0537]

Oh, S., Oh, K., \& Yi, S. K. 2012, ApJS, 198, 4

Ohta, K., Aoki, K., Kawaguchi, T., \& Kiuchi, G. 2007, ApJS, 169, 1

Orban de Xivry, G., Davies, R., Schartmann, M., et al. 2011, MNRAS, 417, 272

Peeples, M. S., \& Martini, P. 2006, ApJ, 652, 1097

Sanders, D. B., Soifer, B. T., Elias, J. H., et al. 1988, ApJ, 325, 74

Sani, E., Lutz, D., Risaliti, G., et al. 2010, MNRAS, 403, 1246

Sani, E., Marconi, A., Hunt, L. K., \& Risaliti, G. 2011, MNRAS, 413, 1479
Satyapal, S., Böker, T., Mcalpine, W., et al. 2009, ApJ, 704, 439

Schawinski, K., Urry, M., Treister, E., et al. 2011, ApJ, 743, L37

Shankar, F. 2009, NewAR, 53, 57

Shankar, F., Salucci, P., Granato, G. L., De Zotti, G., \& Danese, L. 2004, MNRAS, 354, 1020

Shankar, F., Lapi, A., Salucci, P., De Zotti, G., \& Danese, L. 2006, ApJ, 643, 14

Shankar, F., Dai, X., \& Sivakoff, G. R. 2008, ApJ, 687, 859

Shankar, F., Weinberg, D. H., \& Miralda-Escudé, J. 2009, ApJ, 690, 20

Shankar, F., Marulli, F., Bernardi, M., et al. 2012a, MNRAS, submitted [arXiv: 1105.6043$]$

Shankar, F., Weinberg, D. H., \& Miralda-Escude', J. 2012b, MNRAS, submitted [arXiv: 1111.3574]

Shao, L., Lutz, D., Nordon, R., et al. 2010, A\&A, 518, L26

Shlosman, I., Frank, J., \& Begelman, M. C. 1989, Nature, 338, 45

Springel, V., White, S. D. M., Jenkins, A., et al. 2005, Nature, 435, 629

Vika, M., Driver, S. P., Graham, A. W., \& Liske, J. 2009, MNRAS, 400, 1451

Yates, R. M., Kauffmann, G., \& Guo, Q. 2012, MNRAS, accepted [arXiv: 1107.3145]

Watson, L. C., Mathur, S., \& Grupe, D. 2007, AJ, 133, 2435

Woo, J.-H., Treu, T., Barth, A. J., et al. 2010, ApJ, 716, 269 